\title{
Cryptococcal meningitis - a neglected killer
}

In this issue of the SAMJ, Lessells et al. ${ }^{1}$ highlight the unacceptably high mortality due to HIV-associated cryptococcal meningitis (CM) in routine clinical practice in South Africa. CM is now the most common cause of adult meningitis in much of central and southern Africa, accounting for $63 \%$ of all microbiologically confirmed cases in the largest published series. ${ }^{2}$ There are an estimated 720000 cases annually in sub-Saharan Africa, leading to 504000 deaths; ${ }^{3}$ expanding access to antiretroviral therapy (ART) has not yet led to a decline in these numbers. ${ }^{4}$

In research settings in South Africa, utilising optimal amphotericin B-based treatments, acute mortality of CM is between 24 and 37\%.5, Lessells et al. suggest that, in routine care settings, mortality rates are higher. ${ }^{1}$ From their study conducted at Hlabisa Hospital in rural KwaZulu-Natal, they report $41 \%$ in-hospital mortality (all of which deaths occurred within 30 days of admission), which is in keeping with data from Johannesburg, where only $33 \%$ of CM patients were known to be alive and under follow-up at 12 weeks (Govender N, unpublished). Of even greater concern is their finding that only $11 \%$ of CM patients were alive and taking ART at 2 years. Although high early mortality rates are reported in African ART programmes, data from research settings in Cape Town show that, provided patients survive the acute illness, long-term survival of CM patients can be good once they have been established on ART. ${ }^{6}$

The excessive CM mortality in routine care compared with research settings is probably owing in part to patients' difficulties accessing overloaded health care services. This issue almost certainly leads to delays in diagnosis when patients develop CM symptoms, resulting in advanced disease at presentation - a factor consistently identified as a risk for mortality. A further contributor is the lack of availability of amphotericin B (the optimal initial treatment for $\mathrm{CM}$ ) in many hospitals. Outcomes with the alternative, fluconazole monotherapy as initial CM treatment, are poor. ${ }^{7}$ Lessells et al. report that only $35 \%$ of patients in their cohort received any amphotericin $\mathrm{B}$, and only $8 \%$ received what is considered the optimal 2-week course. Unfortunately, this situation appears to be the norm in South Africa, where surveillance data suggest that less than half of patients are receiving optimal initial treatment for CM (even when optimal therapy is defined as a minimum of 1 week of amphotericin or fluconazole $800 \mathrm{mg}$ daily). ${ }^{8}$ An additional critical aspect of the management of CM is correcting elevated CSF pressure (which is present in approximately $75 \%$ of CM cases at presentation ${ }^{9}$ ) with serial lumbar punctures. Guidance for therapeutic lumbar punctures is laid out in the Southern African HIV Clinicians Society CM treatment guidelines, ${ }^{10}$ and requires only basic equipment. But Lessells et al. demonstrate that this simple and important aspect of management is rarely carried out, which corroborates our anecdotal experience.

As Lessells and colleagues highlight, even given these inadequacies in initial treatment, the primary factor accounting for the exceptionally poor long-term outcomes was the loss of almost $50 \%$ of patients to follow-up on discharge from hospital. Only $20 \%$ of patients collected fluconazole secondary prophylaxis, and the majority did not initiate ART. The poor linkage from hospitals to outpatient services and particularly linkage into ART care - is a major problem in South Africa, with many patients lost after inpatient treatment of opportunistic infections such as tuberculosis and CM. This clearly has serious implications, both in terms of short-term outcomes, with high rates of relapse of CM in patients not receiving adequate secondary fluconazole prophylaxis, ${ }^{11,12}$ and also preventing many lives being saved by timely initiation of ART. ${ }^{13}$ Patients with CM and other opportunistic infections involving the CNS represent a particularly vulnerable group in this regard. CNS disease is associated with high mortality and loss to follow-up from ART programmes, which is thought to result from impaired insight, cognition, mobility or volition. ${ }^{14}$ Thirty-four per cent of patients in the Lessells study had a depressed level of consciousness at the time of admission and, even though this may improve during CM treatment, many patients still have impaired cognition during their hospital stay. This impairs patients' ability to comprehend counselling regarding the need for ongoing fluconazole, ART and outpatient follow-up. In addition, our experience suggests that insufficient time is spent counselling patients in this regard in their own language during an admission for CM.

What needs to be done? The good news is that, despite the poor outcomes described in the KwaZulu-Natal cohort, a series of straightforward steps could markedly improve outcomes, even with the currently available treatment options. Clinicians need to have a low threshold for suspecting CM, and all known or suspected HIV-infected patients with a new headache should have a diagnostic lumbar puncture. Amphotericin B should be made available to all clinicians treating such patients, and these clinicians should be educated about the importance of its role in initial therapy, and about the steps needed to monitor for and prevent its adverse effects. Manometers should be available, and clinicians must routinely measure CSF opening pressure in CM patients and manage it according to established guidelines. Importantly, in addition to improving inpatient treatment, subsequent linkage into outpatient ART services must be strengthened. This requires both a streamlining of the referral process with improved communication between inpatient and primary care providers, and specific counselling for patients, emphasising the importance of ongoing fluconazole treatment, the need for ART, and details about where to attend for outpatient care. Involving relatives or friends as treatment supporters during the admission, who could assist with ensuring that such vulnerable patients get to their outpatient appointments, assist with adherence to ART and fluconazole, and bring patients for medical attention if they deteriorate, could make an important difference to outcomes. Health care services must recognise and make provision for the fact that patients who have had an acute opportunistic infection (particularly those that involve the CNS) are vulnerable and need special attention in order to get them onto ART and specifically support them through the first months of treatment. Additional input, both medically and socially, during this early treatment period would pay dividends in terms of long-term survival.

More work is needed to develop simple to use, point-of-care diagnostic tests for $\mathrm{CM}$, to develop improved initial treatments and to define the optimal timing of ART initiation. There is also the exciting possibility that much CM-related morbidity and mortality could be prevented by routine cryptococcal antigen screening of asymptomatic patients entering ART programmes. ${ }^{15}$ But, as Lessells and colleagues clearly demonstrate, CM is exacting a high toll in South African patients now. We can and must do better with the tools we already have.

The Southern African HIV Clinicians Society Guidelines for the Prevention, Diagnosis and Management of Cryptococcal Meningitis are accessible online at: http://sahivsoc.org/index.php?option=com docman\&task $=$ cat_view\&gid $=18 \&$ dir $=$ DESC\&order $=$ date $\&$ Itemid $=67 \&$ limit $=5 \&$ limitstart $=5$ 
JNJ and GM are supported by the Wellcome Trust, London, UK (WT081794 and WT081667).

\section{Joseph Jarvis}

Desmond Tutu HIV Centre, Institute of Infectious Disease and Molecular Medicine, and Division of Infectious Diseases and HIV Medicine, Department of Medicine, University of Cape Town

Infectious Diseases Unit, G F Jooste Hospital, Cape Town

Centre for Infection, Department of Cellular and Molecular Medicine, St George's University of London

\section{Graeme Meintjes}

Division of Infectious Diseases and HIV Medicine, Department of Medicine, and Institute of Infectious Diseases and Molecular Medicine,University of Cape Town Infectious Diseases Unit, G F Jooste Hospital, Cape Town

Department of Medicine, Imperial College, London

Corresponding author: J Jarvis (joejarvis@doctors.net.uk)

\section{References}

1. Lessells RJ, Mutevedzi PC, Heller T, Newell ML. Poor long-term outcomes from cryptococcal meningitis in rural South Africa. S Afr Med J 2011;101:251-252 (this issue).

2. Jarvis JN, Meinties G, Williams A, Brown Y, Crede T, Harrison TS. Adult meningitis in a setting of high HIV and TB prevalence: findings from 4961 suspected cases. BMC Infect Dis 2010;10(1):67.
3. Park BJ, Wannemuehler KA, Marston BJ, Govender N, Pappas PG, Chiller TM. Estimation of the current global burden of cryptococcal meningitis among persons living with HIV/AIDS. AIDS 2009:23(4):525-530.

4. Jarvis JN, Boulle A, Loyse A, et al. High ongoing burden of cryptococcal disease in Africa despite antiretroviral roll out. AIDS $2009231181-118$

5. Bicanic T, Meintjes G, Wood R, et al. Fungal burden, early fungicidal activity, and outcome in cryptococcal meningitis in antiretroviral-naive or antiretroviral-experienced patients treated with amphotericin B or fluconazole. Clin Infect Dis 2007;45(1):76-80.

6. Bicanic T, Wood R, Meintjes G, et al. High-dose amphotericin B with flucytosine for the treatment of cryptococcal meningitis in HIV-infected patients: a randomized trial. Clin Infect Dis 2008;47(1):123130.

7. Jarvis JN, Bicanic T, Harrison TS. Treatment of HIV-associated cryptococcal meningitis in South Africa: the case for amphotericin B over conventional dose fluconazole for initial therapy Southern African Journal of HIV Medicine 2007;28:36-39.

8. Govender N, Cohen C, Meiring S, et al. Trends in Treatment of Adults with Incident Cryptococcosis South Africa, 2005 to 2008. 17th Conference on Retroviruses and Opportunistic Infections, San Francisco; 6 - 19 February, 2010 (abstract 800).

9. Graybill JR, Sobel J, Saag M, et al. Diagnosis and management of increased intracranial pressure in patients with AIDS and cryptococcal meningitis. The NIAID Mycoses Study Group and AIDS in patients with AIDS and cryptococcal meningitis. The NIAID

10. Southern African HIV Clinicians Society. Guidelines for the Prevention, Diagnosis and Management of Cryptococcal Meningitis and Disseminated Cryptococcosis in HIV-infected Patients. Southern African Journal of HIV Medicine 2007;28:25-35.

1. Jarvis JN, Meintjes G, Williams Z, Rebe K, Harrison T. Symptomatic relapse of HIV-associated cryptococcal meningitis in South Africa: the role of inadequate secondary prophylaxis. S Afr Med 2010;100(6):378-382.

12. Collett G, Parrish A. Fluconazole donation and outcomes assessment in cryptococcal meningitis. S Af Med J 2007;97(3):175-176.

13. Jarvis JN, Meinties G, Wood R, Harrison TS. Testing but not treating: missed opportunities and lost lives in the South African antiretroviral therapy programme. AIDS (London, England) 2010:24(8):12331235

14. Asselman V, Thienemann F, Pepper DJ, et al. Central nervous system disorders after starting antiretroviral therapy in South Africa. AIDS 2010:24(18):2871-2876.

15. Jarvis JN, Harrison TS, Govender N, et al, Routine cryptococcal antigen screening for HIV-infected

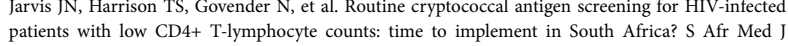
2011:101:232-234 (this issue). 